\title{
Relationship Between ABO Blood Group Phenotypes and nCOVID-19 Susceptibility - a Retrospective Observational Study
}

\author{
Sudhir Bhandari, ${ }^{1}$ Ajit Singh Shaktawat, ${ }^{1}$ Amit Tak, ${ }^{2}$ Jyotsna Shukla, ${ }^{3}$ Jitentdra \\ Gupta, ${ }^{3}$ Bhoopendra Patel, ${ }^{4}$ Shivankan Kakkar, ${ }^{5}$ Amitabh Dube, ${ }^{3}$ Sunita Dia, ${ }^{6}$ \\ Mahendra Dia, ${ }^{7}$ Todd C Wehner ${ }^{7}$
}

\begin{abstract}
Background: Since the outbreak of coronavirus disease-19 (COVID-19) research has been continued to explore multiple facets of the disease. The objective of the present study was to evaluate the relationship between blood group phenotypes and COVID-19 susceptibility.

Methods: In this retrospective observational study 132 hospitalised COVID-19 patients were enrolled from the Swai Man Singh (SMS) Medical Hospital in Jaipur, India after receiving approval from the Institutional ethics committee. The ABO, Rh and Kell blood group phenotypes along with demographic data of the patients were recorded. The observed proportions of 'A', 'B', 'AB', 'O', 'Rh' and 'Kell' blood groups in COVID-19 patients were compared against the expected proportions (the null hypothesis) of the general population using Pearson's Chi-squared test and partition analysis.

Results: There were significant differences between observed and expected frequency for the ABO and Kell blood phenotypes. Further partition analysis of $\mathrm{ABO}$ phenotypes showed that the group ' $\mathrm{A}$ ' phenotypes were more susceptible to COVID-19. The Kell negatives were also more susceptible. The blood groups 'AB', 'B', ' $\mathrm{O}$ ' and ' $\mathrm{Rh}$ ' showed no significant difference for susceptibility to COVID-19.

Conclusion: The study shows a relationship between ABO, Rh and Kell blood groups and COVID-19 susceptibility. The application of these relationships in clinics should be explored in future studies.
\end{abstract}

Key words: ABO blood grouping; COVID-19 susceptibility; Kell blood group phenotypes; Pearson's Chi-squared test; Rh blood group phenotypes.
(1) Department of Medicine, SMS Medical College and Hospitals, Jaipur, Rajasthan, India.

(2) ICMR-National Centre for Disease Informatics and Research, Bengaluru, Karnataka, India.

(3) Department of Physiology, SMS Medical College and Hospitals, Jaipur, Rajasthan, India.

(4) Department of Physiology, Government Medical College, Barmer, Rajasthan, India.

(5) Department of Pharmacology, SMS Medical College and Hospitals, Jaipur, Rajasthan, India.

(6) Department of Rheumatology, Medstar Washington Hospital Center, Washington DC, USA.

(7) Department of Horticultural Science, North Carolina State University, Raleigh, NC, USA.

Correspondence:

AMIT TAK

E: dramitttak@gmail.com

T: +919874472277

\section{ARTICLE INFO}

Received: 3 December 2020 Accepted: 9 December 2020

\section{Introduction}

According to the World Health Organisation report on 16 November 2020, there have been 54,075,995 confirmed cases of COVID-19, including 1,313,919 deaths globally. ${ }^{1}$ The novel coronavirus 2019 (nCOVID-19) has spread beyond the boundaries of Wuhan, China across the world. Researchers in many countries are engaged in discovering associations between
COVID-19 mortality, severity and susceptibility and, other attributes such as demographic, clinical and laboratory findings. ${ }^{2} \mathrm{~A}$ number of diseases have been found associated with various blood group phenotypes.

The ABO blood group antigens are widely distributed throughout the body along with the surface 
of red blood cells. ${ }^{3}$ The association of ABO groups with malaria, pancreatic cancer, duodenal ulcer and other diseases has been elucidated. ${ }^{3-5}$ Microorganisms interact with antibodies against blood group antigens, including ABO, T and Kell systems. ${ }^{6}$ The objective of this study was to evaluate the relationships between the ABO, Rh and Kell blood group systems and susceptibility for the severe acute respiratory syndrome coronavirus 2 (SARS-CoV-2) infection.

\section{Patients and Methods}

A hospital-based, retrospective observational study was designed to evaluate $\mathrm{ABO}, \mathrm{Rh}$ and Kell blood group phenotypes and its relationship to susceptibility to SARS-CoV-2 infection. Laboratory confirmed, real-time reverse transcription polymerase chain reaction (RT-PCR) positive cases of COVID-19, numbering 132, were enrolled in the study from the Swai Man Singh (SMS) Medical College and Hospitals in Jaipur (Rajasthan, India). The hospital provided medical care to patients of various states in North India, including Rajasthan, Harayana, Madhya Pradesh, Uttar Pradesh and Punjab. ${ }^{7,8}$ The mean age of the participants was 36.68 years ( $S D=17.87$ years) and the men to women ratio was 8:3. The ABO, Rh and Kell blood group phenotypes along with demographic data from the COVID-19 patients were collected from laboratory reports. The ABO, Rh (D antigen) and Kell phenotyping was carried out using fully automated solid phase red cell adherence (SPRCA) technology. ${ }^{9}$ The observed frequencies of the blood groups - 'A', 'AB', 'B' and 'O'; 'Rh positive', 'Rh negative'; 'Kell positive' and 'Kell negative' were calculated. The general (expected) propor-

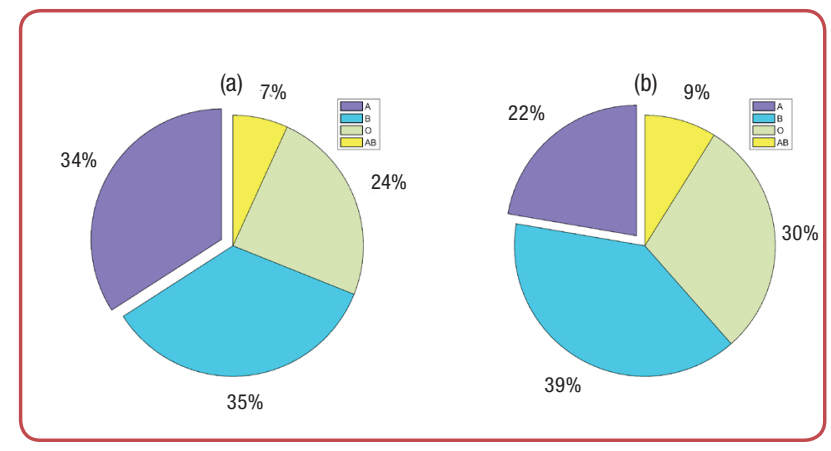

Figure 1: Frequency of $A B O$ blood phenotypes: (a) Observed frequency in COVID-19, (b) Expected frequency in general North Indian population (Garg et al, 2015) tions of the above blood groups were obtained from a study of the North Indian population. ${ }^{10}$ The expected proportions of $\mathrm{ABO}$ blood groups 'A', 'AB', 'B' and 'O' were $22.3 \%, 8.9 \%, 39.2 \%$ and $29.6 \%$, respectively (Figure 1). Rh (D) phenotype were found positive in $93.8 \%$ and negative in 6.2 $\%$. For the Kell system, only $1.6 \%$ were Kell positive, and the remaining were Kell negative. The expected frequencies of the blood group antigens were calculated by multiplying the total number of COVID-19 cases ( $\mathrm{n}=132$ ) with the general proportions of the respective blood groups (Table 1).

Table 1: The observed frequencies of ABO blood group phenotypes in 132 laboratory confirmed COVID-19 patients and expected frequency in general population (Garg et al, 2015).10

\begin{tabular}{|c|c|c|c|c|c|}
\hline & \multicolumn{4}{|c|}{ Blood group phenotypes } & \multirow[b]{2}{*}{ Total } \\
\hline & A & AB & B & 0 & \\
\hline Observed frequency & 45 & 9 & 46 & 32 & 132 \\
\hline $\begin{array}{l}\text { Expected frequency } \\
\text { under } \mathrm{H}_{0}^{*}\end{array}$ & 29.43 & 11.74 & 51.74 & 39.07 & 132 \\
\hline
\end{tabular}

${ }^{{ }^{*} H_{0}}$ is the null hypothesis, which have the expected proportions of $A: A B: B:$ Ophenotypes are $0.22: 0.09: 0.39: 0.30$, respectively.

\section{Statistical analysis}

The observed frequency of blood group phenotypes of ABO, Rh and Kell system were compared against the expected population frequency using Goodness of fit Chi-squared test. The further hypotheses were tested using partition analysis in case of the ABO system. ${ }^{11}$ In order to find the effect of sample size on Type II error, post hoc power analysis was performed. The level of significance was considered at $5 \%$. Software used for the analysis was Jeffreys's Amazing Statistics Program (JASP) (version 0.12.2) software and the Real Statistics package of MS Excel 2010.12,13

\section{Ethical aspects}

The study was conducted in accordance with the Declaration of Helsinki (as revised in 2013). The study was approved by Ethical Committee of the SMS Medical College, Jaipur (No. 416, dated 26 Jun 2020) and individual consent for this retrospective analysis was gained.

\section{Results}

The observed frequencies of $\mathrm{ABO}, \mathrm{Rh}$ and Kell blood group phenotypes with $95 \%$ confidence intervals were calculated (Figure 2). The comparison of the observed and expected frequencies of blood group phenotypes are shown in Figure 3. 


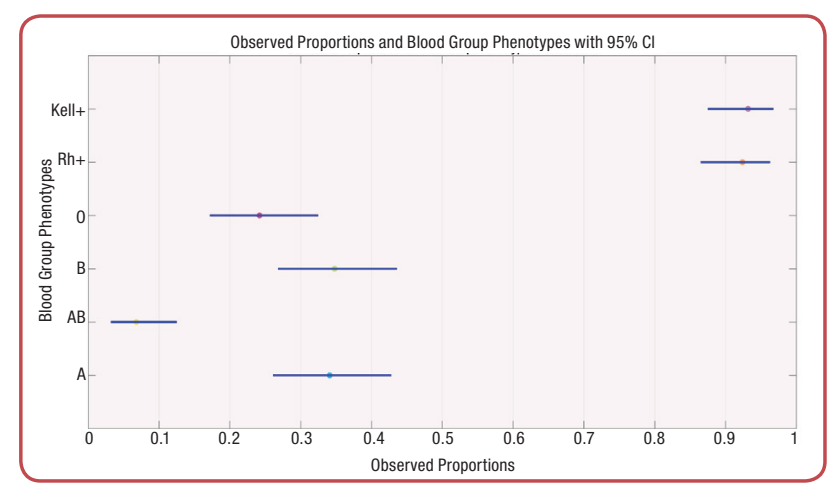

Figure 2: Observed proportions of $A, A B, B, 0$, Rh positive, Kell positive blood group phenotypes with 95\% confidence intervals based on independent binomial distributions of 132 laboratory confirmed COVID-19 patients.

Comparison of phenotypes of $\mathrm{ABO}$ Blood group system for COVID-19 susceptibility

The Goodness of fit of observed and expected frequencies of the $\mathrm{ABO}$ blood groups were tested using Pearson's Chi-squared test (Table 1 and Figure 3).

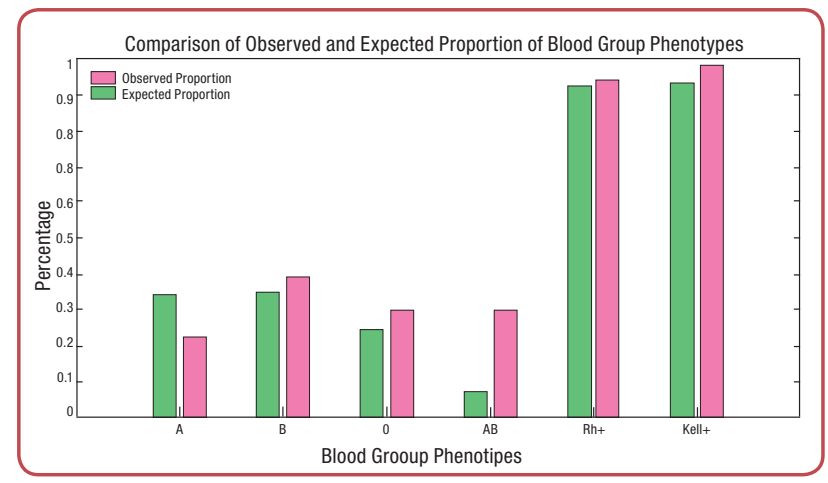

Figure 3: Comparison of observed and expected proportion of blood group phenotypes from a random sample of 132 laboratory confirmed COVID-19 patients and study on North Indian Population (Garg et al, 2015), ${ }^{10}$ respectively.

There were significant differences between expected frequency and observed frequency in at least one of the blood group phenotypes ( $p=$ $0.013)$. The Chi-squared test $\left(\chi^{2}\right)$ does not reveal in which phenotype the difference actually exists. If the observed frequency is less than the expected frequency in one cell, it has to be more in one or the other cells because the total for both the observed and the expected frequencies is the same. The observed frequency was higher in blood group ' $\mathrm{A}$ ' and lower in blood groups 'AB', ' $\mathrm{B}$ ' and ' $\mathrm{O}$ '. In order to confirm the difference in observed frequencies across blood groups, a partition analysis was performed. ABO blood group phenotypes were further divided into two subgroups and two hypotheses were tested using the
Chi-squared test (Table 2). First, the observed and expected frequencies of blood groups 'AB', 'B' and ' $O$ ' were tested and no significant difference was found ( $p=0.89)$. Second, the observed and expected frequencies of blood group ' $A$ ' and 'non- $A$ ' were tested and found to differ significantly $(p=0.001)$. Table 1 was partitioned based on those comparisons and the $\chi^{2}$ test was calculated (Table 2).

It should be noted that the value of $\chi_{\mathrm{T}}{ }^{2}$ is very near to the sum of partitioned $\chi^{2}$ values, as shown in the equation:

$$
\chi_{\mathrm{T}}^{2} \approx \chi_{\mathrm{I}}^{2}+\chi_{\Pi}^{2}=0.23+10.59=10.82
$$

Table 2: Partition of the observed frequencies of $A B O$ blood group phenotypes in 132 laboratory confirmed COVID-19 patients and expected frequency in general population (Garg et al, 2015) (from Table 1) into two sections (Part I and II). Part I includes observed and expected frequencies of $A B$ and $B$ blood groups and part II include observed and expected frequency of ' $A$ ' and ' $O$ ' blood groups.

\begin{tabular}{|c|c|c|c|c|}
\hline & \multicolumn{3}{|c|}{$\frac{\text { Part I }}{\text { Blood group phenotypes }}$} & \multirow{3}{*}{$\begin{array}{l}\text { Total } \\
87\end{array}$} \\
\hline \multirow[b]{2}{*}{ Observed frequency } & $\mathrm{AB}$ & B & 0 & \\
\hline & 9 & 46 & 32 & \\
\hline $\begin{array}{l}\text { Expected frequency } \\
\text { under } \mathrm{H}_{\mathrm{OA}}{ }^{*}\end{array}$ & 9.96 & 43.89 & 33.14 & 87 \\
\hline \multicolumn{5}{|c|}{$\chi_{\mathrm{I}}^{2}=0.23 ;$ degree of freedom $=2 ; p=0.89$} \\
\hline & \multicolumn{3}{|c|}{$\frac{\text { Part II }}{\text { Blood group phenotypes }}$} & \\
\hline Observed frequency & \multicolumn{2}{|c|}{$\begin{array}{c}\mathrm{A} \\
45\end{array}$} & $\frac{\text { Non-A }}{87}$ & $\begin{array}{l}\text { Total } \\
132\end{array}$ \\
\hline $\begin{array}{l}\text { Expected frequency } \\
\text { under } \mathrm{H}_{\mathrm{OB}}{ }^{*}\end{array}$ & \multicolumn{2}{|c|}{29.43} & 102.57 & 132 \\
\hline \multicolumn{5}{|c|}{$\chi_{\Pi}^{2}=10.59 ;$ degree of freedom $=1 ; p=0.001$} \\
\hline
\end{tabular}

Table 3: The observed and expected frequencies of $R h$ antigen system in 132 laboratory confirmed COVID-19 patients.

\begin{tabular}{lcccc}
\hline & \multicolumn{2}{c}{ Rh D antigen system } & \\
\cline { 2 - 3 } & Rh positive & Rh negative & Total \\
\hline Observed frequency & 122 & 10 & 132 \\
\hline $\begin{array}{l}\text { Expected frequency } \\
\text { under } \mathrm{H}_{\mathrm{ORh}}{ }^{*}\end{array}$ & 123.81 & 8.19 & 132 \\
\hline \multicolumn{3}{r}{$\chi_{\mathrm{Rh}}{ }^{2}=0.43$; degree of freedom $=1 ; \mathrm{p}=0.51$} \\
\hline
\end{tabular}

${ }^{*} H_{O R h}$ is the null hypothesis with the expected proportions of $R h$ Positive : Rh Negative phenotypes are $0.94: 0.06$, respectively.

It can be inferred from the above analysis that persons with blood group ' $A$ ' were more susceptible to COVID-19 than 'non-A' groups. Further, there were no significant relationships between the ' $A B$ ', 'B' and ' $\mathrm{O}$ ' blood group phenotypes for susceptibility to COVID-19. 
Comparison of $\mathrm{Rh}$ ( $\mathrm{D}$ antigen) System for COVID-19 susceptibility

The observed and expected frequencies of $\mathrm{Rh}$ blood group phenotypes showed no statistically significant differences $(\mathrm{p}=0.51)$ (Table 3$)$.

Table 4: The observed and expected frequencies of $R h$ antigen system in 132 laboratory confirmed COVID-19 patients.

\begin{tabular}{lccc}
\hline & \multicolumn{2}{c}{ Kell antigen System } & \\
\cline { 2 - 3 } & Kell positive & Kell negative & Total \\
\hline Observed frequency & 123 & 9 & 132 \\
\hline $\begin{array}{l}\text { Expected frequency } \\
\text { under } \mathrm{H}_{\mathrm{OK}}{ }^{*}\end{array}$ & 129.89 & 2.11 & 132 \\
\hline \multicolumn{3}{c}{$\chi_{\mathrm{K}}{ }^{2}=23.83$; degree of freedom $=1 ; \mathrm{p}<0.001$} \\
\hline${ }^{*} \mathrm{HO}_{\mathrm{Ko}}$ is the null hypothesis with the expected proportions of Kell Positive : Kell
\end{tabular}

Negative phenotypes are $0.98: 0.02$, respectively.

Comparison of Kell antigen System for COVID-19 susceptibility

The observed and expected frequencies of Kell blood group phenotypes showed statistically significant differences $(p<0.001)$ (Table 4). The Kell negatives were more susceptible to COVID-19 infection compared to Kell positives.

\section{Discussion}

The blood group antigens are an example of polymorphic traits inherited among individuals and populations. There are 34 recognised human blood groups and hundreds of individual blood group antigens and alleles. The variation in blood group system leads to variations in host susceptibility to many infections. Microorganisms interact with antibodies against blood group antigens, including ABO, T and Kell systems. ${ }^{6}$ The blood group antigens of the $\mathrm{H}, \mathrm{ABO}$, Lewis and historical ' $\mathrm{P}$ ' blood groups contains small carbohydrate epitopes expressed as post-translational modifications on glycoproteins, mucins and glycolipids. The ABO system - International Society of Blood Transfusion (ISBT 001) contains two structurally related carbohydrate antigens, $\mathrm{A}$ and $\mathrm{B}$. The $\mathrm{O}$ or $\mathrm{H}$ antigen is the biosynthetic precursor of $\mathrm{A}$ and $B$ antigens and is classified as a separate blood group system (ISBT 018). All three antigens consist of 2 to 3 terminal oligosaccharides on glycoproteins and glycolipids. ${ }^{14}$ The varying rates of evolution of COVID-19 in two populations may be due to different gene frequency distributions of the blood group and may lead to differences in susceptibility to COVID-19. ${ }^{15}$

In order to evaluate the relationship between
COVID-19 susceptibility and ABO blood group phenotypes, the null hypothesis $\mathrm{H}_{0}$ that the blood group ratio A:B:AB:0 in COVID-19 patients (observed frequency) is exactly the same as the general population (expected frequency) was tested, ie,

$$
\mathrm{H}_{0}: A, A B, B, 0 \text { are in the ratio } 22: 9: 39: 30
$$

The results showed that $\mathrm{H}_{0}$ was rejected (Table 1). The observed frequency of blood group ' $A$ ' was found to be higher than the expected frequency. In addition, observed frequencies in other blood groups were lower than the expected frequencies. If observed frequency is higher than expected frequency in one cell, it has to be lower in one or more of the other cells since the total for both the observed and expected frequencies is the same. Thus, rejecting $\mathrm{H}_{0}$ does not indicate which observed frequency is different from the expected one. In order to check whether the observed frequency of blood group ' $A$ ' is higher than the expected frequency further partition analysis was performed, and two more hypotheses were tested. The corresponding hypotheses were:

$$
\mathrm{H}_{0 \mathrm{~A}}: \mathrm{AB}, \mathrm{B}, \mathrm{O} \text { are in the ratio } 12: 50: 38.2
$$

and

$$
\mathrm{H}_{0 \mathrm{~B}}: \text { A and non-A are in the ratio } 22: 78
$$

The results showed there was no significant difference in observed ratios of AB:B:O blood groups when compared with expected $(\mathrm{p}=0.89)$. However, there was a significant difference in the observed ratio A: non-A blood groups when compared with expected $(p=0.001)$ (Table 2$)$. Thus, it was concluded that blood group ' $A$ ' was more susceptible to COVID-19 than 'non-A' groups. Further there were no significant relationships between the 'AB', 'B' and 'O' blood groups and COVID-19 susceptibility. The power of Chi-squared tests for $\mathrm{H}_{0}, \mathrm{H}_{0 \mathrm{~A}}$ and $\mathrm{H}_{0 \mathrm{~B}}$ hypotheses were $79.5 \%, 6.7 \%$ and $90.2 \%$, respectively. No relationship was found between the Rh (D) antigen and SARS-CoV-2 susceptibility (Power $=10 \%$ ). Also, the Kell negative appeared to be more susceptible to SARS-CoV-2 infection (Power $=99.8 \%$ ). However, exact multinomial test seems to be more accurate. The power analysis showed that type II error was high in hypothesis $\mathrm{H}_{0 \mathrm{~A}}$ and $\mathrm{H}_{\mathrm{Rh}}$ due to the small sample size and conclusions on significance could not be made.

Göker et al investigated ABO blood groups for 186 patients and reported that blood group A (57 \%) was the most frequently detected among the 
COVID-19 patients, followed by the blood group 0 (24.8\%). However, no association between blood group and clinical outcome was established. The Blood group A individuals were significantly higher in number with COVID-19 compared to controls (57 \% vs $38 \%$, p < 0.001; OR: 2.1). While the frequency of blood group 0 was significantly lower in the COVID-19 patients, compared to the control group (24.8\% vs $37.2 \%, \mathrm{p}=0.001$; OR: 1.8) ${ }^{16}$ Likewise, a retrospective cohort study with 265 patients from the Central Hospital of Wuhan showed there was a higher proportion of patients infected with SARS-CoV-2 that have blood group ' $A$ ' than that in healthy controls $(39.3 \%$ vs $32.3 \%$, $p=0.017$ ), while the proportion of blood group ' $\mathrm{O}$ ' in patients infected with SARS-CoV-2 was significantly lower than that in healthy controls $(25.7 \%$ vs $33.8 \%, \mathrm{p}<0.01) .{ }^{17}$ In other recent findings, Ziadi reported a decreased efficiency of adhesion of the Spike protein (S protein) to the angiotensin converting enzyme 2 (ACE2) receptor by antibody $A$, as suggested in other studies. He further stated that a lower susceptibility of blood group 'B' and ' $O$ ' was the case, but it did not explain susceptibility of the 'AB' blood group without anti A and anti B in the blood serum. ${ }^{18}$

In contrast to the above, a study of 397 patients reported an increase in COVID-19 infection associated with the 'AB' blood group. ${ }^{19}$ However, the other findings of Abdollahi ${ }^{19}$ regarding no significant relationship between Rh phenotypes with COVID-19 susceptibility were consistent with the results presented in this study. A study using data from anti-A and anti-B antibodies rather than $\mathrm{ABO}$ blood group reported subjects with anti-A (ie, B and O blood groups) were significantly less susceptible to COVID-19 than those lacking anti-A regardless of group whereas there was no significant difference for anti-B in the serum..$^{20}$ Similarly, Guillon et al and Gustafsson et al argued that either a monoclonal anti-A antibody or natural plasma anti-A present in blood group ' $O$ ' specifically inhibited the SARS-CoV S protein/ ACE2-dependent adhesion to ACE2-expressing cell lines. Therefore, ABO polymorphism could contribute to substantially reducing SARS-CoV transmission. ${ }^{21,22}$ The results from Gustafsson et al confirmed that the glycans have more potential to carry variations than proteins and nucleic acids. The well-known example is polymorphic terminal glycosylation of the ABO blood group family of antigens. The association between infectious diseases and ABO antigens at mucosal surfaces leads to differential adherence of pathogens. Another way the blood group types might affect susceptibility is interaction with the coagulation system. Some factors like von Willebrand factor and factor VIII affect in vivo half-life and clearance of blood type antigens. ${ }^{23}$

Similarly, Dai ${ }^{24}$ studied the interaction among COVID-19 cases, hypertension, and ABO blood grouping. It was reported that in hypertensive patients the renin-angiotensin-aldosterone system (RAS) was overexpressed due to inhibitors of angiotensin converting enzyme (ACE2). The expression of ACE2 receptors was upregulated in hypertensives. The ACE2 receptor was the primary entry site for SARS-CoV-2. Dai mentioned that the ABO blood group was associated with ACE activity and ACE inhibitors induced cough. ${ }^{24}$

The GATC haplotype of the ABO gene polymorphism is prevalent among the non-O blood type patients and is positively associated with an ACE activity. Therefore, 0 blood type carriers have lower ACE levels and are less susceptible to COVID-19 infection. ${ }^{24}$ In a recent genome-wide association study between COVID-19 and disease severity (respiratory failure) 1,980 patients were included from Italy and Spain. Ellinghaus et $\mathrm{al}^{25}$ analysed 8,582,968 single-nucleotide polymorphisms and conducted a meta-analysis of the two case-control panels. They found significant cross-replicating associations with rs11385942 at locus 3p21.31 and with rs657152 at locus $9 q 34.2$. The association signal at locus $9 q 34.2$ coincided with the ABO blood group locus. In a blood-group-specific analysis, blood group A showed higher risk than in other blood groups (odds ratio, 1.45; $95 \%$ CI, 1.20 to 1.75) and blood group 0 showed a protective effect as compared to other blood groups (odds ratio, 0.65; $95 \% \mathrm{CI}$, 0.53 to 0.79$).{ }^{25}$

There may be other mechanisms responsible for higher susceptibility of particular blood group phenotypes that require further studies.

\section{Conclusion}

The study shows a relationship between $\mathrm{ABO}$ blood grouping and COVID-19 susceptibility, with group A being more susceptible. The application of these relationship in clinical practice requires more exploratory studies. 


\section{Limitations of the study}

The expected proportions used in this study lacked information regarding age and sex. Therefore, a multivariate analysis to adjust the effects of the two factors was not possible. However, this may not be necessary as since the distributions of blood group phenotypes were similar across age and gender. The sample size was not enough to provide powerful interpretation of $\mathrm{Rh}$ and Kell blood phenotypes and COVID-19 susceptibility.

\section{Acknowledgements}

None.

\section{Conflict of interest}

None.

14. Cooling L. Carbohydrate blood groups. In: Simon TL, McCullough J, Snyder EL, Solheim BG, Strauss RG, editors. Rossi's Principles of transfusion medicine. 5th ed. New Jersey: John Wiley \& Sons; 2016. p. 159-175.

15. Bhandari S, Shaktawat AS, Tak A, Patel B, Gupta K, et al. A multistate ecological study comparing evolution of cumulative cases (trends) in top eight COVID-19 hit Indian states with regression modeling. Int J Acad Med 2020 Jun;6(2):91-5.

16. Göker H, Aladağ Karakulak E, Demiroğlu H, Ayaz Ceylan ÇM, Büyükaşik Y, Inkaya AÇ, et al. The effects of blood group types on the risk of COVID-19 infection and its clinical outcome. Turk J Med Sci 2020 Jun 4;50(4):679_ 83.

17. Li J, Wang X, Chen J, Cai Y, Deng A, Yang M. Association between $\mathrm{ABO}$ blood groups and risk of SARS-CoV-2 pneumonia. Br J Haematol 2020;190(1):24-27.

18. Zaidi FZ, Zaidi ARZ, Abdullah SM, Zaidi SZA. COVID-19 and the ABO blood group connection. Transfus Apher Sci 2020 Oct;59(5):102838. doi: 10.1016/j.transci.2020.102838.

19. Abdollahi A, Mahmoudi-Aliabadi M, Mehrtash V, Jafarzadeh B, Salehi M. The novel coronavirus SARS-CoV-2 vulnerability association with $\mathrm{ABO} / \mathrm{Rh}$ blood types. Iran J Pathol 2020;15(3):156-160.

20. Gérard C, Maggipinto G, Minon JM. COVID-19 and ABO blood group: another viewpoint. Br J Haematol 2020;190(2):e93-e94. doi:10.1111/bjh.16884

21. Guillon P, Clément M, Sébille V, Rivain J-G, Chou C-F, Ruvoën-Clouet N, et al. Inhibition of the interaction between the SARS-CoV spike protein and its cellular receptor by anti-histo-blood group antibodies. Glycobiology 2008 Dec;18(12):1085-93.

22. Gustafsson K, Durrbach A, Seymour RM, Pomiankowski $\mathrm{A}$. The role of $\mathrm{ABO}$ histo-blood group antigens in viral infections. Trends Glycosci Glycotechnol 2005 Nov;17(98):285-94.

23. Fernandez-Botran GR. ABO blood group type and susceptibility to COVID-19 infection: a brief review. Univ Louisville J Respir Infect 2020;4(1):11. doi: 10.18297/ jri/vol4/iss1/11

24. Dai X. ABO blood group predisposes to COVID-19 severity and cardiovascular diseases. Eur J Prev Cardiol 2020 Sep;27(13):1436-7.

25. Ellinghaus D, Degenhardt F, Bujanda L, Buti M, Albillos A, Invernizzi P, et al. Genomewide association study of severe Covid-19 with respiratory failure. N Engl J Med 2020 Oct 15;383(16):1522-34. 